TIPA. Travaux interdisciplinaires sur la parole et le langage

$31 \mid 2015$

L'impact du contact entre les langues

\title{
Perturbation de la valence des verbes français au contact de l'arabe
}

Perturbation of verbal valence in French as a result of French-Arabic linguistic contact

Cyril Aslanov

\section{(2) OpenEdition}

Journals

Édition électronique

URL : http://journals.openedition.org/tipa/1371

DOI : 10.4000/tipa.1371

ISSN : 2264-7082

Éditeur

Laboratoire Parole et Langage

Référence électronique

Cyril Aslanov, « Perturbation de la valence des verbes français au contact de l'arabe », TIPA. Travaux interdisciplinaires sur la parole et le langage [En ligne], 31 | 2015, mis en ligne le 22 décembre 2015, consulté le 26 mai 2020. URL : http://journals.openedition.org/tipa/1371 ; DOI : https://doi.org/ 10.4000/tipa.1371

Ce document a été généré automatiquement le 26 mai 2020.

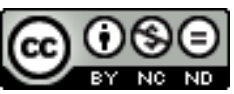

La revue TIPA. Travaux interdisciplinaires sur la parole et le langage est mise à disposition selon les termes de la licence Creative Commons Attribution - Pas d'Utilisation Commerciale - Pas de Modification 4.0 International. 


\title{
Perturbation de la valence des verbes français au contact de l'arabe
}

\author{
Perturbation of verbal valence in French as a result of French-Arabic linguistic
}

contact

Cyril Aslanov

1 Les travaux de Frans van Coetsem sur le rôle du contact linguistique dans le changement diachronique ont bien démontré la corrélation entre la plus ou moins grande stabilité des systèmes linguistiques et leur réceptivité aux influences structurales venues de l'extérieur (Coetsem, $2000: 33,58-60,125)$. Le caractère invasif de la langue interférente affecte non seulement la phonologie ou la morphologie mais aussi la morphosyntaxe, en particulier la valence du verbe. La situation de contact peut transformer un intransitif en transitif ou vice versa ou encore provoquer l'ajout d'un complément prépositionnel non toléré dans les variétés standard de la langue. Du reste, il ne s'agit pas d'une véritable refonte puisque les nouveaux emplois du verbe s'ajoutent à des configurations préexistantes plutôt qu'elles ne viennent les abolir.

2 Le croisement entre le modèle de la valence et la théorie de Coetsem sur la réceptivité à l'influence linguistique lorsque la langue traverse une phase d'instabilité structurelle peut trouver un champ d'application idoine dans les situations de contact entre certains registres du français parlé et certains dialectes arabes. Nous envisagerons diverses situations de contact et d'interférence: le franbanais, variété de français fortement marquée par l'alternance ou le mélange de codes au contact du dialecte arabe libanais; le français d'Égypte, naguère en usage au Caire et en Alexandrie et maintenu vivant par les originaires d'Égypte éparpillés aux quatre coins du monde à la suite des événements de 1956 ; le français pied-noir (pataouète) parlé dans les quartiers populaires des villes de l'Algérie coloniale; le français actuellement pratiqué au Maghreb et chez les Français issus de l'immigration maghrébine.

3 Malgré la diversité des situations de contact envisagées dans le cadre de la présente recherche et en dépit de la variété des dialectes arabes mis en œuvre par ce contact, nous tenterons de proposer un modèle unitaire. De fait, les perturbations de la valence 
constituent un phénomène récurrent dans la plupart des variétés du français au contact de l'arabe. Bien plus, les différences profondes qui séparent les systèmes verbaux de l'arabe et du français amènent à se poser des questions sur le rôle de l'aspect et de la diathèse dans la restructuration de la valence de certains verbes français sous l'influence de l'arabe. Enfin ces analyses ne sauraient faire abstraction de la dimension pragmatique impliquée par les emplois spécifiques des verbes ainsi redéfinis dans leur valence.

\section{Modalités du brouillage de la valence au contact du français et de l'arabe}

\subsection{Les compléments nominaux}

4 Aux yeux d'un puriste normatif, les perturbations de la valence du verbe français dans les variétés du français influencées par un adstrat ou un substrat arabe apparaissent comme des maladresses imputables aux tâtonnements et aux approximations qui caractérisent l'interlangue. Pour les locuteurs du français arabisé, en revanche, ces emplois aberrants sont parfaitement légitimes et consacrés par l'usage parlé. Pour le linguiste, enfin, ces débordements peuvent être considérés comme un enrichissement de la valence de certains verbes du fait de la perméabilité des systèmes linguistiques rapprochés par la situation de contact.

5 On peut classer les modalités de l'enrichissement de la valence de la façon suivante :

a) transitif $\rightarrow$ intransitif (absolutif)

b) complément d'objet externe $\rightarrow$ complément d'objet interne

c) absolutif $\rightarrow$ détermination au moyen d'un complément prépositionnel

6 Par ailleurs, le registre de langue marqué par ces transformations de la valence se caractérise par une réinterprétation du rapport de dérivation entre noms et verbes : à partir d'un substantif les locuteurs créent un verbe dénominatif qui n'existe pas dans la langue normative. Parfois au contraire, le verbe cède la place à une locution verbale employée sans autre complément que la composante nominale de la périphrase verbale.

7 Voici quelques exemples de chacune de ces modalités avec la source probable de l'expression en arabe :

a) transitif $\rightarrow$ intransitif (absolutif)

9 Fréquenter au sens d'entretenir une relation amoureuse en parlant d'un jeune homme ou d'une jeune fille (sans spécification du partenaire). Ce sens n'est pas spécifique aux registres arabisés du français puisqu'on le trouve dans diverses périphéries du monde francophone (Québec; île Maurice) où il constitue probablement la survivance d'un état de langue vieilli (Pan Yan, 2008 : 381). Cela dit, l'adoption de cette formule se justifie en vertu de la perception d'une équivalence entre cet emploi absolutif de fréquenter dans certains registres du français populaire et le verbe dār « tourner » dans son acception spécialisée de dār bi- « tourner autour de; courtiser quelqu'un » en arabe maghrébin. La symétrie n'est certes pas parfaite entre l'expression arabe et son reflet en français régional. Pour des raisons inhérentes à l'acte même de courtiser, le sujet de dār bi- ne peut guère être qu'un homme. Le calque français de l'arabe aurait donc dépassé son 
modèle et transcendé la distinction de genres, si importante dans la société musulmane traditionnelle.

Un autre emploi absolutif concerne le verbe prier, employé non pas au sens global de " se recueillir » mais dans l'acception technique de réciter l'une des prières quotidiennes. En contexte nord-africain, juif aussi bien que musulman, le verbe prier suffit à lui seul à désigner l'accomplissement du rite. Lorsque Michaël Sebban, juif français d'origine algérienne, écrit des phrases comme «je me suis demandé depuis combien de temps personne n'avait prié ici »; «je me suis habillé, j’ai prié et je suis allé prendre un café »; « je prie en regardant les vagues s'écraser sur la plage »(Sebban, 2002:10; 147; 152), il emploie le verbe prier comme d'autres auteurs de facture plus classique auraient employé faire sa prière (Aslanov, 2004: 95). Cette tournure bien implantée dans le français des Juifs pieds-noirs est le calque du verbe șəlla' < arabe classique șalla' " prier ", verbe arabe de la $\mathrm{II}^{\mathrm{e}}$ forme que sa valeur intensive dispense de complément d'objet. De fait, le complément d'objet est implicitement inclus dans ce verbe qui est un dénominatif dérivé du substantif șalah « prière ».

b) complément d'objet externe $\rightarrow>$ complément d'objet interne

prier

13 En français nord-africain, le même verbe prier peut aussi se construire avec le nom de la prière employé comme complément d'objet interne du verbe. En français normatif, l'emploi du verbe prier avec le nom de la prière employé comme complément d'objet interne est un archaïsme qui caractérise le sociolecte des gens d'Église. Le simple fait que l'article soit escamoté devant le nom de la prière récité (prier matines; prier vêpres) signale ces locutions comme des expressions figées remontant au passé de la langue. Mais en contexte musulman ou juif d'Afrique du Nord, l'emploi du verbe prier avec le complément d'objet interne désignant le nom de la prière ne constitue vraisemblablement pas la survivance d'un état de langue ancien. Il s'agit bien plutôt du calque de la construction arabe șəlla' maghrib ou șalla' minhah, respectivement « il a récité la prière du coucher du soleil »; « il a récité la prière de l'après-midi ». Une différence importante sépare l'usage ancien prier matines de ces emplois calquant la syntaxe de l'arabe dialectal nord-africain. Alors que dans le premier cas prier matines est surtout employé à l'infinitif, les francophones musulmans ou juifs d'Afrique du Nord conjuguent le verbe prier accompagné du nom de la prière à tous les temps usuels.

kiffer avec un complément d'objet interne.

15 Ce verbe reproduit en français le verbe de la II forme kayyef/ kiyyəf ou bien le verbe de la $\mathrm{V}^{\mathrm{e}}$ forme tkayyəf/ tkiyyef, notamment lorsqu'ils sont employés sous leur forme participiale de mkiyyəf (II forme) ou de mətkiyyəf ( $\mathrm{V}^{\mathrm{e}}$ forme), comme dans les dialectes marocains (Prémare, 1993-1996: 10, 675-676). Ces formes verbales dénominatives dérivées de kif < turc keyif, nom du chanvre indien, désignaient l'action de fumer du haschisch et par extension l'état de bien-être qui en résulte. En soi-même, cette évolution qui consiste à transformer un verbe exprimant une action en un verbe d'état est un processus remarquable.

Mais l'évolution ne s'arrête pas ici. Au terme d'un développement ultérieur, le verbe kayyef/ kiyyəf « fumer du haschisch; être dans un état de bien-être consécutif à cette consommation " a pu se combiner avec d'autres compléments: ex. ana mkiyyəf had-əlhafla « je prends du plaisir à cette fête». La filière de cette évolution se conçoit aisément : à partir de ana mkiyyəf « je suis dans un état agréable » se sont développés 
des emplois où le mot kif « chanvre » vient expliciter le verbe : ana mkiyyəf (mətkiyyəf) al- kif « je prends du plaisir au chanvre ». À son tour, ce moule syntaxique a été adopté moyennant l'ajout de compléments d'objet direct venant remplacer al- kif « le chanvre " selon un axe de substitution paradigmatique : ana mkiyyəf (mətkiyyəf) had-əl-hafla « je prends du plaisir à cette fête »; ana mkiyyəf (mətkiyyəf) had-əl-mākla « je prends du plaisir à cette nourriture ».

17 Toutefois, d'autres emplois figés révèlent que la valence de kiffer est éminemment complexe. Ainsi, à côté des compléments d'objet interne, on rencontre parfois des pronoms personnels à l'accusatif, comme dans l'expression j'te kiffe; j'le kiffe; j'la kiffe. Généralement, ces pronoms accusatifs sont explicités par des compléments d'objet en position disloquée :j'le kiffe, ce mec; j'la kiffe, cette meuf.

18 Comment analyser ces emplois? S'agit-il de l'extension de la fonction de complément d'objet interne à des objets animés au terme d'une réification de la personne que l'on kiffe? Cette dérive sémantique est tentante, mais une analyse pragmatique des occurrences de j'te kiffe révèle que cette formule s'emploie comme une sorte de déclaration d'amour a priori plutôt que comme une constatation du bien-être qu'on éprouve à filer le parfait amour avec la personne pour laquelle on éprouve un certain penchant.

19 Curieusement, la valeur intransitive de kiffer a été renouvelée par l'emploi de kiffant / kiffante, adjectif verbal issu du participe présent kiffant. La forme kiffant / kiffante est dotée de la valeur causative de "qui provoque le plaisir ». Cet emploi peut s'expliquer de deux façons : soit comme une évolution analogue à celle qui a mené l'adjectif verbal bandant / bandante à revêtir le sens de " qui fait bander ", soit comme un calque du verbe de la II ${ }^{\mathrm{e}}$ forme kayyef employé non pas au sens de " prendre du plaisir ", mais au sens causatif de "faire plaisir à quelqu'un ». Cet usage reflète la répartition des rôles entre la II ${ }^{e}$ forme kayyef et la $\mathrm{V}^{\mathrm{e}}$ forme tkayyef. Alors qu'au Levant, seul kayyef désigne l'action de prendre du plaisir, d'autres dialectes, ceux du Maroc notamment, emploient presque indistinctement la $\mathrm{II}^{\mathrm{e}}$ forme kayyef et la $\mathrm{V}^{\mathrm{e}}$ forme tkayyef au sens de "prendre du plaisir ». Mais dans le Maghreb oriental, on rencontre une spécialisation de kayyef au sens causatif. C'est le cas dans le dialecte Takrouna en Tunisie où la $\mathrm{II}^{\mathrm{e}}$ forme kayyef s'est spécialisée au sens causatif de "faire plaisir ", tandis que la $\mathrm{V}^{\mathrm{e}}$ forme revêt la signification de " consommer du haschisch » et par extension d' éprouver du plaisir » (Marçais-Guiga, 1958-1961 : 35-37).

c) absolutif $\rightarrow$ détermination au moyen d'un complément prépositionnel

voyager employé au sens de "aller à ; se rendre quelque part ».

À la différence des exemples précédents qui se rattachaient à des horizons linguistiques maghrébins, l'emploi de voyager avec un complément de lieu à valeur allative est un arabisme qu'on rencontre dans toutes les variétés du français au contact de l'arabe, de Marrakech à Beyrouth. Cet emploi reproduit la valence du verbe de la III forme sāfara "voyager ». Une phrase comme Il voyagera demain à Beyrouth (traduction-calque de bukra rah ysăfer la-Bairūt) est tout à fait habituelle dans un horizon linguistique arabophone et contraste nettement avec la norme française qui ne tolère pour ce verbe que les compléments de lieu inessifs, c'est-à-dire des locatifs à proprement parler comme dans les phrases : Il voyage dans la région ou Il voyage en Afrique. 


\subsection{Les pronoms compléments et le brouillage entre l'accusatif et le datif} $n i,-k,-u(h),-n a,-k u m$, -hum et les pronoms indirects li, lek, lu, lha, lana, lkum, lhum est abolie en faveur de la première catégorie pour le verbe 'Ațā « donner » qui admet alors un double accusatif (Marçais, 1902: 134). C'est ce qui pourrait expliquer plusieurs emplois aberrants des pronoms ayant une fonction de complément d'objet direct (désormais c.o.d.) le, la, les au lieu du datif ou des compléments prépositionnels avec certains verbes intransitifs en français. Ainsi le verbe șAraH / șraH « hurler » combiné avec le pronom suffixé a été traduit littéralement en « crier quelqu'un ».

24 En vertu du même principe, le verbe se languir de quelqu'un est simplifié en languir quelqu'un sous l'influence du verbe twahḩ̧š qui se construit avec le pronom c.o.d. suffixé : nətwaḩ̧̣̌̌k « je me languis de toi ». Même si des emplois similaires sont attestés en français méridional, le succès de l'expression languir quelqu'un en milieu francophone nord-africain s'explique probablement par une pression du substrat arabe.

25 D'une manière générale, les originaires d'Afrique du Nord ont tendance à étendre l'emploi du pronom c.o.d. à des verbes éminemment intransitifs. Ainsi, le verbe reposer complété par le pronom c.o.d. apparaît-il souvent dans la formule Que Dieu le (la) repose (en paix), substitut consacré de l'exclamation Allah yirhamu (yirhamah) «Que Dieu le (la) prenne en miséricorde ! dans l'horizon francophone maghrébin. Dans ce cas précis, le verbe reposer a été considéré comme un équivalent du verbe rham ( $<$ arabe classique rahama) au terme d'un croisement entre la formule arabe musulmane citée ci-dessus et la formule catholique qu'il (elle) repose en paix, traduction du latin requiescat in pace. En outre un facteur paronomastique a pu jouer un rôle additionnel dans ce recyclage du verbe reposer en-dehors de sa valence normale. De fait, le verbe rahama « avoir pitié de » ressemble vaguement au mot rāhah « repos » et cette ressemblance a pu fonctionner comme une passerelle de transition entre les deux constructions.

\subsection{Du complexe au simple et du simple au complexe}

Le verbe pèleriner employé par les francophones marocains - musulmans et juifs reproduit tous les emplois du verbe zār « se rendre sur la tombe d'un santon ». Ce n'est pas à proprement parler un néologisme puisqu'en français, le verbe pèleriner est attesté depuis le XIVe siècle. Toutefois sa réactivation en français marocain reflète la volonté de restaurer une série étymologiquement cohérente entre le verbe $z \bar{a} r$, le participe present $z \bar{a}$ 'ir et le nom d'action ziārah " pèlerinage ». Certes le rapport est inversé puisque en arabe, le verbe zār est la forme première par rapport à laquelle $z \bar{a}$ ir et ziārah sont des dérivés, tandis qu'en français, le verbe pèleriner est secondaire par rapport à pèlerin. Néanmoins, le fait même que le verbe simple pèleriner ait été exhumé du passé de la langue (peut-être pas tout à fait sciemment) révèle qu'au contact avec l'arabe, le français a été sommé de fournir un équivalent simple et synthétique de zār.

27 Inversement, il arrive qu'au contact de l'arabe nord-africain, le français des jeunes issus de l'émigration maghrébine remplace un verbe simple par une périphrase verbale. Ainsi les verbes haïr et détester sont remplacés par la locution avoir la haine contre. Et pourtant, l'arabe dialectal possède le verbe simple krah (arabe classique kariha) pour

TIPA. Travaux interdisciplinaires sur la parole et le langage, 31 | 2015 
exprimer ce sentiment. Pour expliquer ce développement, il faut sans doute invoquer des motivations inhérentes à chacune des deux langues en contact. Tout d'abord, le paradigme du verbe haïr en français est d'un maniement difficile du fait de l'alternance déroutante entre le thème hai- $d u$ singulier et le thème haïss- du pluriel (en cela haïr constitue une exception notable au sein de la classe morphologique des verbes du II ${ }^{\mathrm{e}}$ groupe). L'emploi du verbe détester aurait assurément pu pallier ces inconvénients, mais il a sans doute été perçu comme plus faible sémantiquement que haïr. C'est ce qui expliquerait l'emploi de la locution avoir la haine contre où le nom d'action haine rappelle par sa présence ce verbe doublement irrégulier dont l'usage est si problématique. Or cette explication interne peut être complétée utilement par une considération touchant à l'interférence de l'arabe dialectal maghrébin en contact avec le français. En Afrique $\mathrm{du}$ Nord on entend souvent des phrases comme fiya kərha 'al dont j'ai la haine contre semble bien le calque. Bien plus, cette formule connaît une variante qui se substitue à elle selon l'axe paradigmatique. Il s'agit de la phrase j'ai le seum contre (parfois francisée en j'ai la rage contre) qui transpose fiya səmm ‘al, littéralement « j'ai le poison contre ; j’ai la colère contre ». Non seulement, la pression de l'arabe a permis de substituer une locution absolutive aux verbes transitifs haïr et détester, mais en plus, ces formules analytiques s'insèrent dans un réseau cohérent où elles peuvent aisément permuter comme les formules arabes dont elles constituent l'écho.

Dans ce chassé-croisé entre les locutions aller en pèlerinage ou accomplir un pèlerinage remplacées par pèleriner et les verbes simples haïr ou détester auxquels se substituent les formules analytiques j'ai la haine contre, j'ai la rage contre, j'ai le seum contre on perçoit une asymétrie. En effet, le remplacement de la locution analytique par un verbe synthétique implique le remplacement d'une valence présentant un complément d'objet interne par une valence strictement intransitive qui ne tolère que des circonstants. En revanche, le démantèlement des verbes haïr et détester qui sont des transitifs de plein droit ne constitue pas une transition de la transitivité à l'intransitivité puisqu'aussi bien haine, rage et seum sont des compléments d'objet interne du verbe avoir.

\section{Implications aspectuelles et pragmatiques des perturbations de la valence}

Bien que la question de l'aspect ne soit pas directement liée à celle de la valence, les transformations de la valence provoquées par le contact avec l'arabe ont rejailli sur la configuration aspectuelle des emplois verbaux calqués sur la phraséologie de la langue en contact. Des emplois comme tu m'as saoûlé(e), il m'a tué(e) (au sens positif de « tu m'as fait mourir de rire ») ou je t'ai langui(e) reproduisent littéralement les expressions sakkertni «tu m'as saoûlé » (littéralement " tu m'as fermé »); qtalni «il m'a tué »; tuhḥaštək/ tuḥhaštik « je me suis langui de toi » respectivement. Cette valeur de présent dévolue au parfait est tout à fait normale dans une langue sémitique où la distinction aspectuelle entre le perfectif et l'imperfectif prévaut sur les oppositions temporelles. Une fois transposées en français, ces expressions risquent de prêter à confusion quand elles sont proférées en-dehors de la communauté linguistique en contact direct ou indirect avec l'arabe. Mais pour les locuteurs de l'arabe ou pour ceux dont le français est influencé par un substrat arabe, il est clair que l'action exprimée par le verbe ou 
l'état qui en résulte ne font pas référence à un passé révolu, mais à une situation encore valide au présent.

À cela s'ajoute peut-être une valeur évidentielle qui dans d'autres états de langue ou dans d'autres langues aurait été exprimée par des moyens syntaxiques ou lexicaux (comme la particule ö́p en grec ancien) plutôt que proprement morphologiques. En français plus hexagonal, c'est le présent renforcé par une tournure exclamative qui est employé pour souligner la force du sentiment exprimé: (qu'est-) ce que tu peux m'embêter! ou (qu'est-) ce que tu peux être c... au lieu de tu m'as saoûlé(e); tu me fais rire là ! au lieu de tu m'as tué(e) !; (qu'est-) ce que tu me manques ! au lieu je t'ai langui(e)!

31 Quant à la dimension pragmatique, elle est essentielle pour la compréhension de ces énoncés. En l'absence d'informations sur la situation de l'énoncé, la phrase il m'a tué(e)! apparaît comme une entorse flagrante à la logique sauf à considérer qu'on fasse parler un revenant. Mais quand il est prononcé sur un ton hilare et avec une touche d'accent maghrébin ou pied-noir, cet énoncé prend tout son sens.

32 Outre les dimensions aspectuelle et pragmatique qu'ils impliquent, ces énoncés présentent une particularité qui intéresse directement la valence. En effet, les sujets et les compléments d'objet qu'ils recèlent figurent presque toujours à l'état de pronoms. Des phrases comme Bébert a saoûlé mémé ou Momo a tué Khadidja dans lesquels les verbes s'entendent à double sens risquent fort d'être pris au pied de la lettre si les satellites pronominaux du noyau verbal sont remplacés par des noms de plein droit. Le cas de languir est plus complexe, car il n'exprime que le sentiment de nostalgie. Par conséquent, une phrase comme Les élèves ont langui leurs professeurs est envisageable. Mais des énoncés comme Bébert a saoûlé mémé ou Momo a tué Khadidja sont susceptibles d'être interprétées comme Bébert a enivré mémé ou Momo a assassiné Khadidja. En revanche, les constructions disloquées permettent de maintenir l'effet pragmatique et aspectuel de la construction faisant intervenir des pronoms : Bébert, il l'a saoulée, à mémé ; Momo, il l'a tuée, à Khadidja notez le remplacement du complément d'objet direct par un complément prépositionnel).

33 Cette intéressante restriction de la valeur figurée de saoûler et tuer aux constructions pronominales à l'exclusion des constructions faisant intervenir des noms est sans doute due au fait que ces expressions sont employées dans le feu de l'échange verbal, c'est-àdire dans une situation où les principes de l'intersubjectivité décrits par Émile Benveniste rendent nécessaire le recours aux pronoms plutôt qu'aux noms (Benveniste, 1966). Dans le tourniquet intersubjectif des identités déléguées à ces jokers que sont les pronoms, même la $3^{\text {e }}$ personne est susceptible d'assumer le rôle de substitut universel. Cela se vérifie en particulier à propos de l'expression il (elle) m’a tué(e) qui peut s'employer à propos d'un interlocuteur présent dont on souhaite tourner les propos en ridicule en prenant à témoin d'autres personnes présentes ou absentes et en recourant à l'hyperbole consistant à employer le verbe «tuer » au sens figuré de «scandaliser » ou « étonner».

34 Ainsi donc, ces calques syntaxiques de l'arabe ne perturbent pas la valence en tant que telle mais le statut des actants et des valents ainsi que les implications aspectuelles et sémantiques des énoncés. Le fait que ceux-ci ne soient compatibles qu'avec des actants et des valents pronominalisés pourrait être lié au critère de saturation. En effet, les emplois figurés de saoûler et tuer sont incompatibles avec des actants ou des valents de plein droit, pour des raisons qui sont directement dues au palimpseste arabe qui leur est sous-jacent. Les expressions arabes correspondantes consistent en un seul mot, le 
verbe, suffixé au moyen des pronoms compléments d'objet : sakkertni « tu m'as saoûlé(e) »; qtalni « il m'a tué(e) » tuhhašstək/ tuhhaštik « je t'ai langui(e)». Quant à l'actant sujet, il n'est évident pas exprimé comme il est normal dans les langues prodrop dont l'arabe fait partie. Pour rendre la concision de l'expression arabe constituée d'un seul mot en tout et pour tout, le français qui s'en fait l'écho se devait donc de limiter les actants et les valents aux satellites immédiats du noyau verbal, ce qui exclut les substantifs.

35 Enfin, l'impact combiné de la valence et de l'aspect arabe sur le français se manifeste aussi sans rapport avec l'emploi aspectuel/ évidentiel du passé. L'une des manifestations de ce genre d'interférence concerne l'emploi itératif du présent avec des verbes qui ne s'emploient guère dans cette acception en français métropolitain. Un jeune Égyptien francophone disait naguère à propos de ses activités dans un country club des bords du Nil : Nous nous retrouvons là-bas; nous buvons du jus d'orange; nous nous aimons. Dans ce contexte, l'expression nous nous aimons, calque de me-nhebb ba'd ne désignait pas la situation psychologique de l'amour mutuel, mais la description itérative de flirts occasionnels dans la jeunesse dorée cairote.

\section{De la pragmatique à la sociolinguistique}

Nous avons signalé dans la partie précédente que les énoncés comme "tu m'as saoûlé(e) »; « il m'a tué(e) », « je t'ai langui(e) » ne font sens qu'à la condition d'être insérés dans un discours connoté comme maghrébin ou pied-noir. Le filtrage de la signification qui permet de comprendre qu'on a affaire à un sens figuré et expressif et non au sens littéral est donc dépendant de la situation de parole et de l'insertion du locuteur à l'intérieur de son discours ethnique. Il est intéressant de comparer d'un point de vue sociolinguistique les implications de l'emploi de ces formules si connotées. S'agit-il d'un pur ethnolecte ou bien peut-on considérer qu'au-delà de la connivence ethnique, une dimension d'exhibitionnisme identitaire entre également en ligne de compte?

37 La réponse à cette question est complexe, d'autant qu'elle implique deux communautés linguistiques: les jeunes Français musulmans issus de l'émigration maghrébine et les Juifs d'Afrique du Nord originaires d'Algérie ou de Tunisie, les Juifs marocains constituant un cas à part pour des raisons que j'ai expliquées en d'autres lieux (Aslanov, 2002). Pour la première catégorie, le substrat arabe dialectal est encore assez proche, tandis que pour la seconde, la langue ancestrale est un souvenir plus ou moins lointain : moins dans le cas des Juifs tunisiens, plus pour les Juifs pieds-noirs qui dans certaines régions d'Algérie comme Alger et l'arrière-pays algérois avaient abandonné leur parler judéo-arabe dès les deux premières décennies du XXe siècle (Cohen, 1912 : 10-12). On se rappelle les réflexions nostalgiques de Jacques Derrida déplorant cette éradication de la (ou des) langue(s) ancestrale(s) (Derrida, 1996: 63-74). De par sa naissance dans une bourgade du Département d'Alger (El Biar), le jeune Juif pied-noir avait été coupé de la langue que parlaient encore ses parents.

38 De sorte que les jeunes Français musulmans utilisent des calques qu'ils peuvent rattacher à la langue de leurs parents (dans la mesure où leur connaissance de cette dernière est préservée). De leur côté, les Juifs algériens et tunisiens, jeunes ou moins jeunes, emploient des expressions dont ils ne perçoivent pas toujours l'origine arabe. Le critère de l'âge est important, car les vieilles générations de l'immigration maghrébine 
se meuvent dans un horizon bilingue, tandis que leurs descendants sont des monolingues ayant de fortes réminiscences de l'arabe qui, pour eux, est au moins un quasilecte (sur ce terme, voir Glinert, 1993). Chez les Juifs d'origine maghrébine, en revanche, le monolinguisme renforcé par une connaissance quasilectale de l'arabe concerne presque uniquement les vieilles générations. La connaissance que leurs petits enfants ont préservée de la langue ancestrale se réduit à quelques bribes, souvent déformées et réinterprétées à l'aune de la phonétique française.

39 Or les emplois décrits ci-dessus ont beau calquer des structures arabes, ils n'en demeurent pas moins du français. On comprend mieux dès lors les avantages stratégiques de ces tournures françaises arabisées. Elles constitueraient en quelque sorte un analogon de la langue érodée et réduite au statut de quasilecte dans le meilleur des cas. Il se crée donc un décalage entre les rythmes d'évolution des identités ethnique et linguistique - celle-ci en phase de disparition et celle-là toujours vivace. Pour compenser cette faille entre les deux identités, la langue de l'ex-colonisateur se voit investie de la mission d'exprimer l'identité ethnique que l'arabe quasilectal ou quasiment oublié, n'est plus en mesure d'assumer.

40 L'intérêt pragmatique du maintien de ces valences perturbées apparaîtra mieux si l'on s'avise du fait que c'est la trace fossilisée d'une langue dont la connaissance s'est peu ou prou perdue dans le contexte assimilateur de la France jacobine, capable de laminer les identités linguistiques sans pour autant éradiquer les identités ethniques. L'arabe dialectal maghrébin apparaît comme la langue de pépé et mémé pour les Juifs d'origine nord-africaine ou bien la langue du deblé pour les jeunes beurs. En revanche les registres du français émaillés de marqueurs culturels maghrébins sont auréolés d'un coefficient de prestige. En effet, leurs usagers revendiquent leur différence face aux générations précédentes qui parlaient mal le français ou qui ne parlaient pas uniquement cette langue. Ils se démarquent en outre du français des « céfrans » ou des " patos » par cette saveur nord-africaine préservée tantôt par inertie, tantôt au terme d'un processus conscient qui dans certains cas s'apparente à un maniérisme dandy (indication of coolness, dans la terminologie sociolinguistique anglo-saxonne). Rappelons en effet qu'à l'ère du rap, ces variétés maghrébines du français jouissent d'une certaine vogue en-dehors même des milieux où elles sont pratiquées spontanément.

41 Au Liban, la situation est bien entendu très différente, car la connaissance de l'arabe levantin a été préservée intacte même dans les milieux de la diaspora si l'on fait exception des communautés syro-libanaises implantées aux États-Unis ou au Brésil depuis la fin du XIX ${ }^{e}$ siècle. Mieux encore, dans ces milieux arabes chrétiens du Levant dispersés loin de la patrie historique, la préservation du français est bien souvent le corollaire du maintien de l'arabophonie. Les deux langues sont apparemment si imbriquées que la disparition de l'une entraîne l'érosion de l'autre. On est loin du schéma de la substitution de l'arabe par le français puisque dans ces situations, c'est l'anglais (aux Etats-Unis et en Australie) et le portugais (au Brésil) qui renvoient dos à dos les deux langues collatérales pour reprendre le concept naguère développé par Jean-Michel Éloy (Éloy, 2004).

42 Dans ces conditions, les perturbations de la valence de certains verbes ne sont pas dues à l'impact d'un substrat oublié, mais à l'interférence d'une langue que les locuteurs emploient en alternance avec la variété levantine du français. L'alternance de code entre le français et l'arabe a donc influé sur les structures mêmes du français libanais (Gueunier, 1993 ; Aslanov, 2006 : 214-219). Les emplois comme il voyage à Beyrouth au 
sens de « il va à Beyrouth » ou nous nous aimons au sens de « nous vivons des amourettes » (cas relevé en Égypte) s'intègrent dans un continuum où le français arabisé alterne avec l'arabe libanais ou égyptien.

\section{Conclusion}

43 Au terme de ce passage en revue des conséquences du contact entre le français et diverses variétés de l'arabe dialectal, il apparaît que les nombreux calques syntaxiques arabes en français ont exercé une onde de choc sur les emplois de certains verbes d'usage courant. S'agit-il pour autant d'une perturbation? L'analyse et le classement des faits ont révélé qu'au-delà de la valence à proprement parler, d'autres dimensions entrent en jeu: l'aspect; la pragmatique et bien entendu, la sociolinguistique. Mais curieusement, ces emplois n'ont pas annulé les usages plus normatifs des verbes en question. Le cas de tu m'as tué ou de tu m'as saoûlé est particulièrement révélateur de ce mécanisme à deux vitesses qui s'instaure dans les variétés du français exposé à l'interférence du substrat ou de l'adstrat arabes. Plutôt que d'une perturbation qui aurait porté atteinte aux œuvres vives de la langue, il faudrait parler d'un enrichissement ou d'un élargissement des possibilités expressives de la langue moyennant un assouplissement des structures héritées. Des phénomènes analogues sont attestés dans d'autres zones de contact entre le français et des langues allogènes. Il n'est que de penser aux variétés du français parlées en Wallonie ou en Suisse romande où l'on reconnaît des interférences syntaxiques du néerlandais et de l'allemand, respectivement.

44 L'assouplissement de la valence des verbes dans ces variétés du français en contact avec d'autres langues est susceptible de remettre en question certains présupposés théoriques concernant l'actance et la valence des verbes. Le modèle traditionnel instauré par Heinz Happ (Happ, 1976) doit prendre en compte le facteur variationniste qui se fait jour dans l'interface entre les langues. L'étude du contact entre le français et l'arabe révèle que la valence des verbes est une donnée ouverte, susceptible d'être modifiée selon les aléas de la diachronie, de la diatopie et de la diastratie.

45 Enfin, les usages étudiés dans ce chapitre soulèvent la question de l'interface entre syntaxe et sémantique. Les variations dans la valence font certes intervenir des paramètres syntaxiques tels que l'absence ou la présence de tel ou tel complément ou bien la transformation d'une locution en un verbe synthétique ou vice versa. Mais les conséquences ultimes de ces perturbations de la valence touchent en fait à la sémantique puisqu'elles relèvent de la dynamique du calque interlinguistique. L'assouplissement de la valence est aussi une façon d'étendre la palette de significations d'un verbe en le faisant coïncider avec celle du verbe correspondant dans la langue en contact, en l'occurrence l'arabe. 


\section{BIBLIOGRAPHIE}

Aslanov, C. (2002). Le français des Juifs d'Afrique du Nord est-il un judéo-français ? Perspectives : Revue de l'Université hébraïque de Jérusalem 9, p. 79-101.

Aslanov, C. (2004). La Terre promise, pas encore... de Michaël Sebban ou la création d'un 'vulgaire illustre' judéo-français. Humoresques (Rires marginaux, rires rebelles), 19, p. 93-101.

Aslanov, C. (2006). Le français au Levant, jadis et naguère : à la recherche d'une langue perdue, Paris: Honoré Champion.

Benveniste, É. (1966). La nature des pronoms, Problèmes de linguistique générale, I, p. 251-257.

Coetsem, F. van. (2000). A General and Unified Theory of the Transmission Process in Language Contact, Heidelberg: Carl Winter.

Cohen, M. (1912). Le parler arabe des juifs d'Alger, Paris: Champion.

Derrida, J. (1996). Le monolinguisme de l'autre, Paris: Galilée.

Éloy, J.-M., (2004) « Des langues collatérales: problèmes et propositions », dans: Jean-Michel Éloy (éd.), Des langues collatérales : problèmes linguistiques, socio-linguistiques et glottopolitiques de la proximité linguistique, Paris: L'Harmattan, I, p. 5-25.

Glinert, L. (1993). « Language as Quasilect: Hebrew in Contemporary Anglo-Jewry », dans: Lewis Glinert (éd.), Hebrew in Ashkenaz: A Language in Exile, Oxford, Oxford University Press, p. 249-264.

Gueunier N. (1993). « Les Francophones du Liban: 'Fous des langues' », dans: Didier de Robillard et Michel Beniamino (éd.), Le Français dans l'espace francophone. Description linguistique et sociolinguistique de la francophonie (tome I), Paris: Honoré Champion, p. 263-279.

Happ, H. (1976). Grundfragen einer Dependenz-Grammatik des Lateinischen, Göttingen: Vandenhoek \& Ruprecht.

Marçais, W. (1902). Le dialecte arabe parlé à Tlemcen: grammaire, textes et glossaire, Paris: Ernest Leroux.

Marçais, W. \& Guiga, A. (1958-1961). Textes arabes de Takroûna - Glossaire, Paris: Librairie orientaliste Paul Geuthner.

Pan Yan, C. (2008). « Les régionalismes du français mauricien dans la presse francophone mauricienne (de 1777 à nos jours) », dans: André Thibault (éd.), Richesses du français et géographie linguistique, Bruxelles: De Boeck Université, II, 317-452.

Prémare, A.-L. de. (1993-1996). Langue et culture marocaine. Dictionnaire arabe-français, Paris: L'Harmattan.

Sebban, M. (2002). La Terre promise, pas encore..., Paris: Ramsay.

\section{RÉSUMÉS}

Cet article passe en revue les conséquences que le contact du français avec divers dialectes arabes (maghrébins; levantin) a pu avoir sur la valence de certains verbes. Les modifications de la valence des verbes français au contact de l'arabe apparaissent comme la systématisation de calques syntaxiques favorisés par des situations d'alternance ou de mélange de codes entre le 
français et quelques dialectes arabes (maghrébins, égyptien et levantin). Sur le plan de l'analyse syntaxique les modifications apportées à la valence du verbe peut être perçue comme une perturbation. Néanmoins il apparaît que le changement de valence implique d'autres dimensions tant sur le plan morphosyntactique (aspect et Aktionsart) qu'au niveau pragmatique et sociolinguistique. Une approche variationiste révèle que le contact avec l'arabe ne provoque pas le remplacement d'une structure par une autre, mais permet l'enrichissement des possibilités expressives de tel ou tel verbe ou de telle ou telle locution verbale. On assiste donc à la coexistence entre une structure non-marquée du point de vue aspectuel ou pragmatique avec des structures fortement marquées (exemple: tu m'as tué au sens de "tu me fais rire " ou tu m'as saoûlé au sens de "tu m'ennuies»). Cet article s'intéresse aussi aux constructions éliptiques consistant à employer un verbe avec une valeur absolutive sur le modèle de constructions similaires en arabe dialectal. Ces considérations amènent à relativiser la pertinence du modèle de la valence promu par Heinz Happ à l'étude des variétés de langue fortement marquées par le contact et l'hybridation.

This article is a survey of the main consequences resulting from the contact between French and several Arabic dialects as far as verbal valence is concerned. The impact exerted by Arabic dialects on the valence of French verbs appears as a systematization of occasional syntactical calques as a result of Arabic-French code-switching or code-mixing. On the level of syntactic analysis the change of valence as a result of linguistic contact may be perceived as a perturbation. However, it appears that the change of valence involves several other dimensions either on a morphosyntactical level (aspect and Aktionsart) or from the view point of pragmatic and sociolinguistic.

Interestingly enough, those specific uses of the verbs coexist with more standard uses thereof. The cases of tu m'as tué, literally "you killed me", in the sense of "you must be kidding", or tu m'as saoûlé, literally "you fuddled me", in the sense of "you piss me off" are typical of a two-tiered mechanism within the varieties of French exposed to the interference of an Arabic substrate or adstrate. Here the aspectual and pragmatic values involved in the use of the passé composé (present perfect) prevent from understanding the verbs tuer and saouler in the literary meaning of "killing" or "fuddling".

Thus the coexistence of an unmarked construction with a marked one is not so much a perturbing factor that allegedly affected the very structure of the language but rather an enrichment or a widening of the expressive potential of French thanks to the bestowing of a greater deal of elasticity to the inherited system or to the standard. Similar phenomena are attested in other zones of contact between French and other languages. Let us think for instance of the varieties of French in use in Wallonia or French Switzerland where syntactic inteference from Dutch and German respectively are recognizable in verbal constructions.

The bestowing of more elasticity to verbal valence in the varieties of French in contact with other languages can help reassess some theoretical presuppositions regarding morphosyntactic alignment and valence. The traditional model promoted by Heinz Happ should take into account the variationist factor that comes to the foreground in the interface between two languages in contact. The study of the contact between French and Arabic reveals that verbal valence is perhaps more elastic than it appears in Happ's theory. The great deal of variations that can affect verbal valence according to diatopic, diachronic and diastratic factors is something that Happ did not sufficiently take into consideration, may be because his theory is mainly founded on corpora of Latin literat texts, i.e. on a highly standardized linguistic variety.

Lastly, the deviant uses of verbs or verbal constructions studied in this article raise the question of the interface between syntax and semantic. The variation of valence involves syntactical parameters like the skiping of a complement from a given verbal phrase (absolutive use of an otherwise transitive verb), or conversely, the addition of a complement to a verb normally 
considered as intransitive. It can also involve the transformation of a verbal phrase into a single verb or alternatively, the creation of a verbal periphrase usually centered around the verb avoir. However, the ultimate consequences of those syntactical transformations are clearly pertaining to semantics because they sometimes involve considerable differences on the level of expressivity besides their being the product of calque translation from Arabic. Whatever the distinctions that should be made between the syntactical, the semantic and the pragmatic dimension, the widening of the valence is a way to enrich the gamut of significations of French verbs according to the gamut of significations of their counterparts in Arabic dialects: the Lebanese dialect as far as franbanais, the Lebanese blend of French is concerned; Egyptian as regards Jews born in Pre-Nasserian Egypt; various Maghrebi dialects when it goes on varieties of French in decolonized North Africa or in post-colonial France.

INDEX

Mots-clés : contact français-arabe, variétés du français hors de France, dialectes arabes (maghrébins, égyptien, levantin), franbanais, valence verbale, transitivité, locutions verbales Keywords : French and Arabic in contact, varieties of French outside France, Arabic dialects (Maghrebi, Egyptian, Levantine), franbanais, verbal valence, transitivity, verbal periphrases

\section{AUTEUR}

\section{CYRIL ASLANOV}

Aix Marseille Université, CNRS, LPL UMR 7309, 13100, Aix-en-Provence, France

msaslan@mail.huji.ac.il 